\title{
Localization of the Best Measuring Point for Gearwheel Behaviour Testing Using Group of Adaptive Models Evolution
}

\author{
A. Dočekal ${ }^{1}$, V. Dynybyl ${ }^{2}$, M. Kreidl ${ }^{1}$, R. Šmíd ${ }^{1}$, P. Žák ${ }^{2}$ \\ ${ }^{1}$ Department of Measurement, Faculty of Electrical Engineering, Czech Technical University in Prague, \\ Technicka, 2, 166 27, Prague 6, Czech Republic, e-mail: docekaa@fel.cvut.cz, kreidl@fel.cvut.cz, smid@fel.cvut.cz \\ ${ }^{2}$ Department of Production Machines and Mechanics, Faculty of Mechanical Engineering, Czech Technical University in \\ Prague, Technicka, 2, 166 07, Prague 6, Czech Republic, e-mail: Vojtech.Dynybyl@fs.cvut.cz, Pavel.Zak@fs.cvut.cz
}

\begin{abstract}
This paper deals with signal processing for vibration analysis of the gear transmission. The main goal was localization of the best measuring point on the gearbox housing, which could provide the highest sensitivity to gearwheels vibration. Vibration measured at some selected points on the gearbox housing was analysed using methods of spectral analysis. The important frequencies in the vibration spectra and a value of their importance were estimated using Group of Adaptive Models Evolution. This work was done within the framework of experimental research concerned with testing and lifetime estimation of new designs of gearwheels. The lifetime of an examined gearwheel, which is mainly related to rise of pitting faults, was assessed by monitoring of a gearwheel vibration level.
\end{abstract}

Keywords: Spectral analysis, vibration, gearbox, gearwheel behaviour, Inductive Modelling, Group of Adaptive Models Evolution

\section{INTRODUCTION}

REQUIREMENTS to ensure a longer lifetime of gearwheels in modern rotating machinery, e.g. in modern designs of automotive gearboxes, contribute to research into new designs of used gearwheels. Some new designs of gearwheels are especially focused on various tooth face geometry, material and heat processing.

Beside Acoustic Emission, a fault of a gearwheel, e.g. pitting, may also be detected in early stage by vibration monitoring of the gear transmission. Measurement of the gearwheel vibration enables to track the fault evolution too. Due to high financial requirements or another technological issues, it is difficult or even impossible to measure vibration directly at gear transmission in some parts of gearboxes, so vibrations are often measured on gearbox housing. Localization of the best measuring point on the gearbox housing, which could provide the highest sensitivity to gearwheels vibration, was the objective of our approach.

\section{SUBJECT \& METHODS}

\section{A. Testing Stand}

A universal testing stand was used to test gearwheels. The stand is designed to abridge the lifetime of gearwheels. The design of Niemann's closed loop circuit [1] was used for this purpose because of its lower energy intensiveness. The testing stand, shown in Fig. 1 and Fig. 2, consists of one measured gearbox and one auxiliary gearbox, a drive engine, tension equipment, and sensors of twisting moment, revolution rate, temperature and vibration.

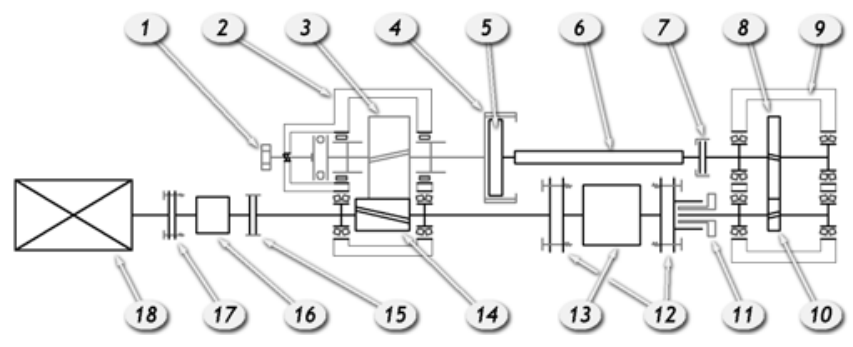

Fig. 1 Nieman's closed loop circuit

1 - Tensing screw, 2 - Auxiliary gearbox, 3 - Movement able gearwheel, 4 - Movement able part of gear coupling, 5 - Fixes part of gear coupling, 6 - Torsional shaft, 7 - Coupling with evolvent grooving, 8 - Tested gearwheel, 9 - Testing gearbox, 10 - Testing pinion, 11 - ETP-Techno coupling, 12 - Radex-N coupling with cut able screw, 13 - Moment sensor, 14 - Axial fixed pinion, 15 - Radex-N coupling with cut able screw, 16 - Moment sensor, 17 - Radex-N coupling, 18 - Drive engine

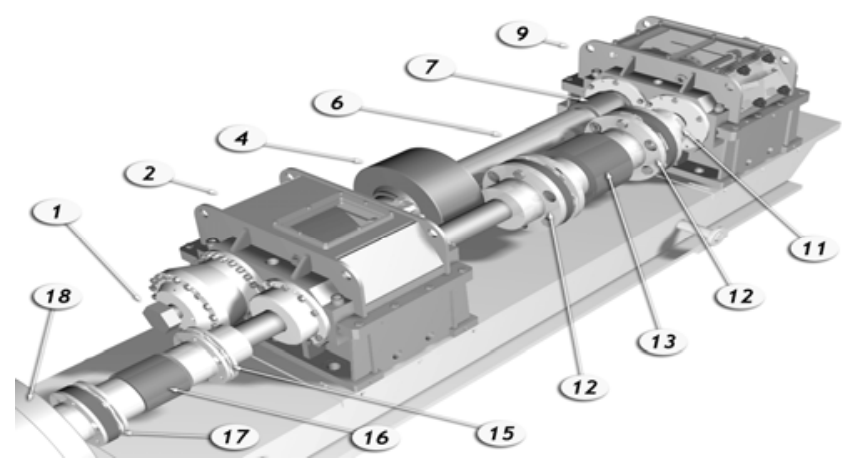

Fig. 2 The testing circuit visualization

The design of the testing stand utilizes a pair of gear transmissions which are geared in order to operate in parallel run. The tensing screw (see Fig. 1) enables to apply a certain 
value of the twist moment loading the gearwheels under test.

Gearwheels testing conditions [2] and their mounting should be similar to actual operational conditions, so gearwheels were tested while being under a defined load or, more precisely, under a pre-selected twist moment. However, it is necessary to shorten the time of the test and so the load above operational limits was applied to the tested gear transmission. In our case, we were limited by using the moment sensor up to $5000 \mathrm{Nm}$. The circuit is dimensioned for maximal virtual power $785 \mathrm{~kW}$ and for revolution rate of 1450 RPM.

\section{B. Data Acquisition and Sensor Placing}

Vibrations generated by gear transmissions are transferred through a shaft and rolling bearing into the gearbox housing. These vibrations mixed with noise are measured on outer surfaces of a gearbox housing.

During the tests performed at the tested gearbox, four places were estimated to be convenient for placing the accelerometers for gearing vibration measurement with a view to assumed propagation of the gearing vibrations. The highest gearing vibration level and lower undesirable effects as vibrations of plates could be assumed at these points. The selected points are located at bearing housing above the shafts close to the rolling bearings. They are shown in Fig. 3. The selected four different points on the housing form a basic group of sensor placing, from which one place has to be selected by an analysis described below. Vibrations were measured at three orthogonal directions shown in Fig. 3. Each point is denoted by a number ( 1 to 4 ) and a letter denoting the direction (A - axial, $\mathrm{H}$ - horizontal, V - vertical).

The vibrations were measured by Brüel \& Kjœr PULSE 7537 analyzer fitted with calibrated accelerometers 4507 B. The placing of the accelerometers is shown in Fig. 4. The used attachment of accelerometers enables to operate up to the upper limit frequency at $3 \mathrm{kHz}$ with sensitivity $10 \mathrm{mV} / \mathrm{ms}^{-2}$.

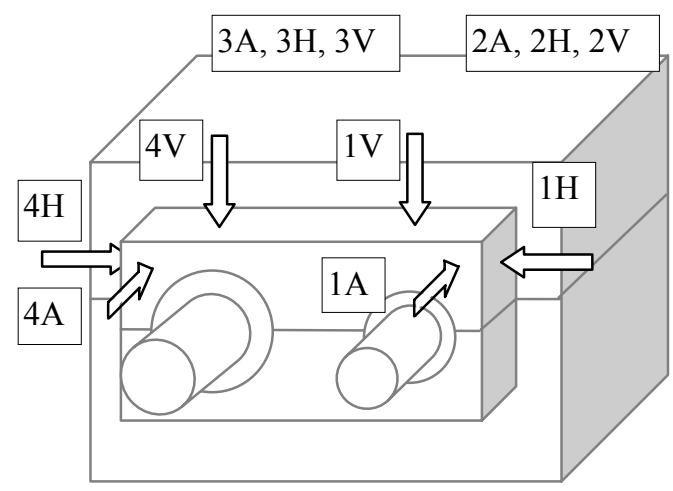

Fig. 3 Points and directions tested on the gearbox

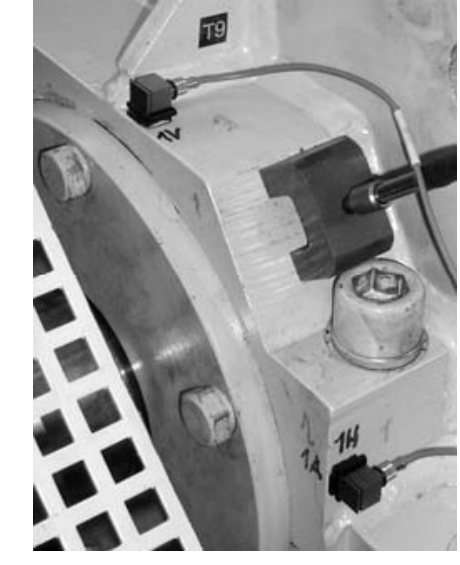

Fig. 4 Accelerometers placing on the gearbox housing

\section{Digital Signal Processing}

Beyond the minimal level of noise and background vibration, the best accelerometer placing point can be described by the ability of the measured vibration to represent conditions of the analysed gear transmission in the best way. The vibration signal measured on the best placing point at the appropriate direction has to offer the best sensitivity to change the gear transmission state. Within this context, the problem of finding the best placing point can be assigned to the problem of the parameter estimation.

An important presumption of this process states that there exists a parameter which can be changed and its change primarily involves vibrations of the gear transmission. As described in section 2.A, the analysed gear transmissions were tested using the universal testing stand where different predefined load values (torque moments) were applied to the gear transmission during the experiments in order to obtain a vibration signal containing vibration of the transmission at different working states. The vibration signals measured at different load values were used instead of the different gearing operational status because of the expensiveness of this solution.

Because of the presence of background noise and vibration it is vital to apply spectral analysis for the localization of the best accelerometer placing point [3]. In particular, the Power Spectral Density (PSD) of vibration signals was used. For each accelerometer placing point and each direction it is necessary to find out a few frequency bands in spectra most involved by the load value applied to the gear transmission. Then the extent of the load influence to the vibration level can be evaluated in these bands only.

The selection of a few frequency bands, which are most important for gear transmission vibration analysis, is complicated and requires mutual comparison of many vibration spectra. This comparison can be done visually - by comparing the vibration spectra via cascade diagram (shown 
in Fig. 6). Therefore it is convenient to simplify and automate this process by application of any appropriate feature extraction method. This paper is focused on using Group of Adaptive Models Evolution (GAME), in the described project. Group of Adaptive Models Evolution represents a suitable method from a set of Inductive Modelling methods.

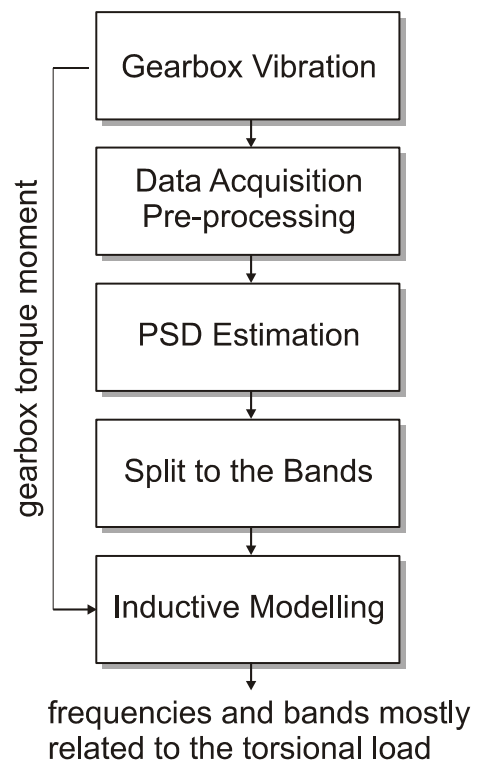

Fig. 5 Vibration spectral analysis using Inductive Modelling

The spectral analysis using Inductive Modelling can be described by the block diagram shown in Fig. 5. The vibration signals measured at all placing points and at all directions, including the actual value of the torque load applied are recorded. The value of the applied torque load is a part of each record. The signals are pre-processed in accordance with filter noise and useless part of spectra is omitted from the analysis. Then Power Spectral Densities of vibration signals are estimated. Welch's method was used for this purpose. The Power Spectral Densities are split into bands whereas the level of vibration within each band is expressed by its total power. The set of all measurements is divided into the training and verifying part. GAME Inductive Modelling method is applied to the sets in order to recognize the most frequently involved bands by the actual value of torque load.

\section{1) Spectral Analysis}

Vibrations measured at the pre-selected points on the gearbox housing were analysed, using methods of spectral analysis [1]. This approach enables to recognize vibration caused by the gear transmission. In the vibration spectrum, the conditions of the gear transmission can be recognized by evaluating the vibration PSD at Tooth Frequency (TF) and Hunting Tooth Frequency (HTF).
The Tooth Frequency $\left(f_{T}\right)$ is given by (1).

$$
f_{T}=f_{1} \cdot n_{1}=f_{2} \cdot n_{2}
$$

where $f_{1}, n_{1}$ are a revolution rate and a number of teeth of the larger gearwheel and $f_{2}, n_{2}$ are a revolution rate and number of teeth of the smaller gearwheel.

Hunting Tooth Frequency $\left(f_{H T}\right)$ can be expressed by (2).

$$
f_{H T}=\frac{f_{t}}{n_{1} \cdot n_{2}} N_{C D},
$$

where $N_{C D}$ is the smallest common divider of teeth numbers for both gearwheels.

Both frequencies mentioned above and their higher harmonic and subharmonic frequencies are characteristic for certain gear transmission and revolution rate and their amplitude modulation by shafts revolution rates can describe actual gear transmission condition. The evaluation of the levels of vibration at these frequencies and their changes can enable to find out any changes in the gear transmission operational status, among others especially the wear of gearing or occurrence and evaluation of a fault in the gear transmission.

\section{2) Group of Adaptive Models Evolution}

Frequencies which are most often involved with the operational status of the gear transmission could be found using methods of Inductive Modelling. The Group of Adaptive Models Evolution (GAME) was used to recognize

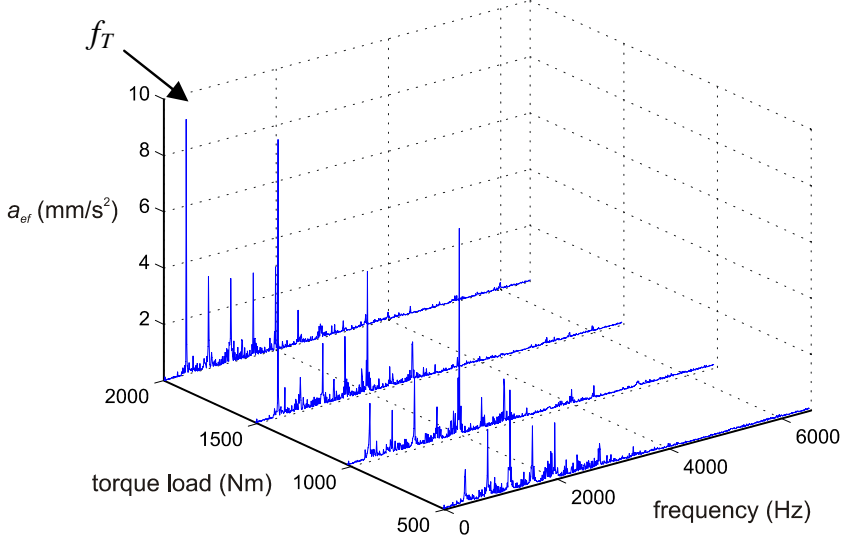

Fig. 6 Cascade diagram showing dependency of vibration spectra at the measuring point denoted by $2 \mathrm{H}$ to the gear engagement load

frequencies important for gear transmission analysis in this project. The inputs of GAME were the equidistantly divided bands. GAME selected the bands which contain considerable information about dependency of the vibration levels at TF and HTF frequencies on the gear engagement load.

Inductive Modelling methods, such as Group of Adaptive 
Models Evolution [4] [5] use machine learning techniques to derive models directly from the measured data in spite of methods of deductive modelling as back-propagation or RBF neural networks, which use domain expertise to fit predefined math models of a system to the measured data. Inductive Modelling is factually based on sorting out gradually complicated models and selection of the best solution by a minimum of external criterion. This leads to the selection of the features which can describe the real problem in the best way.

\section{RESULTS}

The data measured in this experiment contained 5 classes formed by different states of the torque forcing the gear transmission. The measured vibration data set contained 120 records: 15 records for the torque at $0 \mathrm{Nm}, 15$ records for 500 $\mathrm{Nm}, 30$ records for $1000 \mathrm{Nm}, 30$ records for $1500 \mathrm{Nm}$, and 30 records for $2000 \mathrm{Nm}$. The records order was jumbled using a random process with normal distribution.

Tooth Frequency $f_{T}$ characteristic for the tested gear transmission is $398 \mathrm{~Hz}$. The bands that were mostly rated by GAME are as follows: $f_{T}, 2 f_{T}, 5 f_{T}, 7 f_{T}$. The overview of dependency of the total effective value of the vibration acceleration within the band for first 6 bands on the gear transmission torque load is shown in Fig. 7.

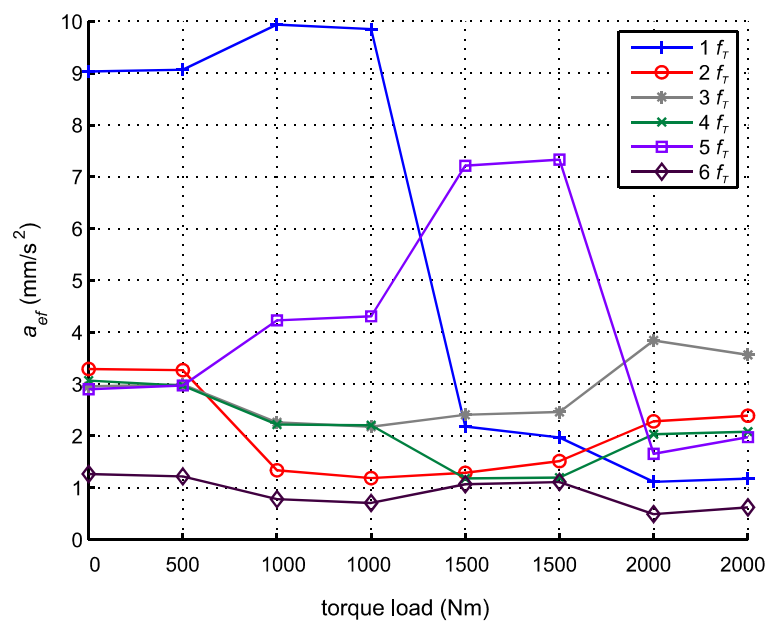

Fig. 7 Dependency of the effective value of total vibration within each band on the gear transmission torque load at the measuring point denoted by $2 \mathrm{H}$

The evaluation of vibrations only at selected frequencies showed that the highest level of gearing vibration can be generally found in horizontal orientation of the accelerometer sensitivity direction. The most promising point denoted as $2 \mathrm{H}$ seems to be located at the gear transmission housing above the low-speed shaft. The measurements at this point do not markedly show ineligible parasitic vibrations of coupling and oscillations of gearbox plates.
Subsequent visual examination of spectra of vibrations, which were measured at different pre-selected points on the gearbox housing, proved that the measuring point selected by our approach and subsequent analysis seems to be the best point for gearing vibration measurement in the view of dependency of the vibration spectra on the gearing load too.

\section{CONCLUSION}

This paper is focused on the usage of the Group of Adaptive Models Evolution for an automated recognition of the frequencies or bands which are mostly related to gear transmission condition monitoring.

The proposed method of extraction of the most important features from the measured vibration data was applied to find out the best accelerometer placing point on the tested gearbox housing for the testing and lifetime estimation of new designs of gearwheels and gear transmission.

Because of the project setting, the conditions of the gear transmission were evaluated using Power Spectral Density of the measured vibration signals. The automatic evaluation of the measured vibration spectra, using Group of Adaptive Models Evolution, can be also applied in the case when different spectral transformations are used in order to describe the gearing vibrations in a suitable way.

\section{ACKNOWLEDGMENT}

The research was supported by the research program No.MSM6840770015 "Research of Methods and Systems for Measurement of Physical Quantities and Measured Data Processing" of the CTU in Prague sponsored by the Ministry of Education, Youth and Sports of the Czech Republic.

\section{REFERENCES}

[1] Dynybyl, V., Dočekal, A., Žák, P. (2006). Experimental research of gearwheels behaviour. Acta Mechanica Slovaca 10(4-B), 119-124, ISSN 1335-2393.

[2] Dynybyl, V., Mossóczy, P., Češpíro, Z. (2005). Experimental assessment of bevel gearboxes. In 22nd Danubia-Adria Symposium on Experimental Methods in Solid Mechanics, September 28 - October 12005 (pp. 296-297). Parma: University of Parma.

[3] Kreidl, M. et al. (2001). Diagnostic systems. Prague: Czech Technical University Publishing House, 352 p., ISBN 80-01-02349-4.

[4] Kordík, P., Šnorek, M. (2007). Progress in inductive modelling. In EUROSIM, 6-10 September 2004. Vienna: EUROSIM-FRANCOSIM-ARGESIM, CD-ROM.

[5] Kordík, P. (2006). Fully automated knowledge extraction using group of adaptive models evolution. $\mathrm{PhD}$ thesis, FEE, Czech Technical University in Prague. 